\title{
Understanding the nature and outcome of childhood
} wheezing

\section{To the Editors:}

The recent study by MATRICARDI et al. [1] from the Multicentre Allergy Study (MAS) cohort provides useful confirmation of existing understanding about the origins of asthma. Thus, they highlight the early roots of much childhood wheeze and the good prognosis of not wheezing in early life. Their confirmation of the heterogeneity of childhood wheeze is also welcome and complements our similar work from the Isle of Wight Birth Cohort [2]. They find common ground too with us on the importance of atopic family history and atopic sensitisation in predicting persistence of wheeze [3] in children with earlyonset wheeze. However, we wish to discuss some of their main findings.

MATRICARDI et al. [1] restrict their discussion of predictive "risk scores" for outcome of early-life wheezing to the Tucson Index. We draw their attention to our own score, published 5 yrs ago in this journal [3]. It comprises four factors: family history of asthma; recurrent chest infections in infancy; absence of nasal symptoms at age 2 yrs; and atopic sensitisation at age 4 yrs. When all four were present, there was a positive predictive value of 83.3 and a negative predictive value of 63.9 for predicting persistence of early-life wheezing up to the age of 10 yrs. That score is at least a starting point in developing a clinically relevant predictive tool. Testing it on a different population such as the MAS cohort would have been worthwhile and it is a pity that an opportunity to do that has been missed. Future collaboration between numerous research groups could provide meaningful answers to vexing questions such as "the outcome of early-life wheezing".

The identification of risk factors for different wheezing phenotypes is important. When assessing risk factors in our cohort for development of early persistent childhood wheeze compared to never wheezing, we found somewhat different results to those of MATRICARDI et al. [1]. Unlike them, we found that in addition to inherited atopy-related factors, environmental factors such as parental smoking, recurrent chest infections in infancy and lower social grouping were risk factors for development of that phenotype [4]. It is surprising to see a lack of environmental influence in the MAS study findings. Study design may be important in that regard. Our sample was an unselected whole-population cohort, whereas the MAS cohort incorporated a subgroup deemed to be at high genetic risk of atopy. It is plausible that in the presence of overwhelming genetic predisposition for asthma and atopy such influences outweigh those of any environmental factor. Thus, the wider utility of their findings needs recognition. It is also worth noting that their "study subpopulation" was more likely to have nonsmoking parents and also to have been breastfed than the overall MAS cohort. These findings might explain the lack of environmental effects such as tobacco smoke and raise the possibility of unaccounted-for effects of social class, which might also have influenced results. Previously, we found in our cohort [5] that mothers of lower social class tended to smoke more and breastfeed less, all of which may have a complex influence on asthma risk.

The study of MATRICARDI et al. [1] addresses another taxing question: that of predicting which nonwheezers in early life will develop later wheeze. We previously [2] showed that such late-onset wheezers were a clinically significant group. Our findings [4] for risk factors for that phenotype showed a lack of association with environment that is consistent with those from the MAS cohort. Based on our findings for early persistent and late-onset wheeze, we proposed that while genetic predisposition towards developing asthma is crucial for wheezing phenotypes throughout childhood, environmental exposures in early life may facilitate earlier disease expression. Confirmation of such thinking is awaited but we recently highlighted [6] some of the intricate interactions of tobacco smoke and genetic polymorphism that might predispose to early persistent wheeze in our cohort. In the same issue of this journal as the study of MATRICARDI et al. [1], BOTTEMA et al. [7] nicely demonstrated that the interplay of gene and environment is highly complex and intriguingly suggested that timing of environmental exposure may be paramount.

MATRICARDI et al. [1] serve a timely reminder of the diversity and complexity of childhood wheeze. However, the wider applicability of their findings remains to be seen and the role of environment in asthma pathogenesis merits further study. Indeed as expertise advances, the study of gene-environment relationships in wheeze and asthma development is entering an exciting new phase. As Sherlock Holmes [8] might say: "Come, Watson, come, the game is afoot!"

\section{Scott, R.J. Kurukulaaratchy, A. Raza and S.H. Arshad} David Hide Asthma and Allergy Research Centre, St Mary's Hospital, Newport, Isle of Wight, UK.

\section{STATEMENT OF INTEREST}

None declared.

\section{REFERENCES}

1 Matricardi PM, Illi S, Gruber C et al, Wheezing in childhood: incidence, longitudinal patterns and factors predicting persistence. Eur Respir J 2008; 32: 585-592. 
2 Kurukulaaratchy RJ, Fenn MH, Waterhouse LM, Matthews SM, Holgate ST, Arshad SH. Characterization of wheezing phenotypes in the first 10 years of life. Clin Exp Allergy 2003; 33: 573-578.

3 Kurukulaaratchy RJ, Matthews S, Holgate ST, Arshad SH. Predicting persistent disease among children who wheeze during early life. Eur Respir J 2003; 22: 767-771.

4 Kurukulaaratchy RJ, Matthews S, Arshad SH. Does environment mediate earlier onset of the persistent childhood asthma phenotype? Pediatrics 2004; 113: 345-350.

5 Arshad SH, Hide DW. Effects of environmental factors on the development of allergic disorders in infancy. J Allergy Clin Immunol 1992; 90: 235-241.
6 Sadeghnejad A, Karmaus W, Arshad SH Kurukulaaratchy R, Huebner M, Ewart S. IL-13 gene polymorphisms modify the effect of exposure to tobacco smoke on persistent wheeze and asthma of childhood. A longitudinal study. Respir Res 2008; 9: 2.

7 Bottema RWB, Reijmerink NE, Kerkhof M, et al. Interleukin 13, CD14, pet and tobacco smoke influence atopy in three Dutch cohorts: the allergenic study. Eur Respir J 2008; 32: 593-602.

8 Conan Doyle A. The Return of Sherlock Holmes. London, George Newnes Ltd, 1905.

DOI: $10.1183 / 09031936.00149408$

\section{Pre-immigration screening for pulmonary tuberculosis}

\section{From the authors:}

The authors thank BHUNIYA [1] for his interesting comments on our article published in the European Respiratory Journal (ERJ) [2]. Tuberculosis (TB) screening policies are influenced by the structure of the national TB programme, the existing financial resources, the expertise of available professionals and the sense of apprehension expressed by the local population in the host country.

The aim of our study was to evaluate the unique screening process performed in Ethiopian immigrants arriving in Israel, and to share our experience with the ERJ's readers.

The tuberculin skin test (TST) is considered the preferred method for screening latent TB infection (LTBI) in immigrants originating from countries with high rates of TB [3-5]. This method can potentially detect all patients with LTBI [6] in a simple, inexpensive and sensitive fashion. The merits of this instrument are increased when the physician considers the patients' age, previous TB exposure and past BCG vaccination in addition to the TST results. The interpretation of the TST using those additional data increases the specificity of the test [7].

Chest radiography (CXR) is considered the preferred test for the immediate detection of radiological findings suggestive of active pulmonary TB (PTB) [8]. The reliability of CXR depends on the experience and interpretative skills of the reader [9], knowledge of the sex and HIV status of the patient [10] and the quality of the film. In addition, it can detect abnormalities compatible with previous TB infection. Therefore, some experts recommend this instrument for screening immigrants originating from high-TB-incidence countries for LTBI [11].

As for the interpretations of the CXR, readers should be aware that the same team consists of three radiologists, who are working in the same hospital and have performed this task for nearly $7 \mathrm{yrs}$, while decreasing their inter-observer variability. As for the individual risk of radiation while performing CXR, the literature does not see or prove risk to the diagnosed patient if it is performed within the indication of screening or future treatment. It is also known that CXR is not commonly performed in Ethiopia for this low-income and rural people, and the clinical diagnosis prevails. Thus, in most cases, this is the immigrant's first life-time exposure to radiography.

Most industrialised countries use a combination of CXR and TST to screen immigrants originating from high-TB-incidence countries [12-15]. The Israeli TB screening policy in Addis Ababa, Ethiopia includes both of these methods for the detection of LTBI and PTB. The Israeli screening process employs additional measures to support those instruments and to augment the validity of the entire process, such as physical examination and symptom questionnaire for each immigrant. In addition, the follow-up is resumed while the immigrants are housed in the designated absorption centres and are tested for the second-step TST. Medical follow-up is provided by a nurse at these centres during the first few years of acclimatisation in Israel.

HIV tests are performed for the Ethiopian immigrants only after arrival in Israel. Israeli immigration laws are unique, and allow immigrants of Jewish descent to be naturalised in Israel upon arrival, and provided with all social benefits including national health insurance. Therefore, HIV-infected immigrants are entitled to follow-up and therapy when indicated. The right to immigrate to Israel is unrelated to the health status of the immigrants, and there is high social sensitivity in Israel to any bureaucratic barriers for the homecoming of Jews. However, because HIV may stigmatise this a priori marginalised population of immigrants, we prefer to perform HIV testing in Israel in a non-coercive manner only after arrival. This way, the immigrants are reassured that no pre-arrival medical condition deprives them of the anticipated homecoming. We do, however, test for TB prior to immigration, as the mycobacterium may infect otherwise healthy individuals during air travel to Israel and immediately thereafter. The results of TB screening performed previously in Ethiopia are compared with the HIV tests conducted in Israel to compose a comprehensive evaluation of each individual.

Finally, isoniazid resistance was diagnosed in less than $5 \%$ of the PTB immigrants. Therefore, we assume that the relatively low isoniazid resistance and the relatively low risk for sideeffects of the therapy, as well as the high cost-effectiveness of 